\title{
New Photometric Surveys of the Fornax Dwarf Spheroidal Galaxy $^{1}$
}

\author{
T.A. Smecker-Hane ${ }^{2}$ \\ Dept. of Physics \& Astronomy, 4129 Reines Hall, Univ. of California, \\ Irvine, $C A$ 92697-4575, USA \\ P.B. Stetson, J.E. Hesser \\ National Res. Council of Canada, Herzberg Inst. of Astrophysics, \\ Dominion Astrophysical Obs., Victoria, BC V8X 4M6, Canada
}

\begin{abstract}
We report on our new photometric surveys of star fields in the Fornax dwarf spheroidal (dSph) galaxy designed to investigate its star formation and chemical evolution histories.
\end{abstract}

\section{Introduction}

At a Galactocentric distance of $130 \mathrm{kpc}$ and $M_{V}=-13.7 \mathrm{mag}$, Fornax is among the most distant and luminous of the remarkably diverse family of gas-poor dSph galaxies that orbit the Milky Way. We have obtained color-magnitude diagrams (CMDs) as input to a comprehensive study of the star-formation rate and chemical evolution of the Fornax dSph. The evolution of Fornax, and most other dSph companions of the Milky Way, was much more complex than previously envisioned. By investigating dSphs, we can place powerful constraints on parameters of sophisticated theoretical models of galaxy evolution and understand the roles that massive dark matter halos, photoionization and explosions of massive stars, and galactic winds have in shaping galaxy evolution.

\section{Our Wide-Field Photometric Survey}

The results of our wide-field photometric survey of bright stars in the Fornax $\mathrm{dSph}$, obtained with the CTIO 1.5-m telescope, were recently published (Stetson et al. 1998). This survey spanned an area of $\sim 0.33 \mathrm{deg}^{2}$ around the center of the Fornax dSph. For reference, the core and tidal radii of the Fornax dSph are $13.7^{\prime}$ and $71^{\prime}$, respectively (Irwin \& Hatzidimitriou 1995). Thus our survey included most of the galaxy. Although the primary goal of the survey was to identify red giants for subsequent spectroscopy and chemical abundance determination, we also used the data to show that the spatial distributions of different-aged stellar populations in Fornax are not identical.

\footnotetext{
${ }^{1}$ Based on observations made with the Cerro Tololo Inter-American Observatory. CTIO is operated by AURA, Inc. under cooperative agreement with the National Science Foundation.

${ }^{2}$ Partial funding provided by NSF grant AST-9619460 to TSH.
} 
We identified variable stars in the well-observed inner fields as probable RR Lyraes and used them to trace the old ( $\sim 15 \mathrm{Gyr})$ population (see fig. 11 of Stetson et al. 1998; also fig. 3 of the paper by Grebel \& Stetson in this volume). Although we cannot accurately define their core radius, we can tell that the old stars are the least spatially concentrated of Fornax's stellar populations. Based on their location in the CMD, we identified red clump (core-helium burning) stars as tracers of the intermediate-aged (3-10 Gyr) population, and bright blue main-sequence stars as tracers of a young ( $\lesssim 400 \mathrm{Myr}$ ) population. The very young stars have a significantly different spatial distribution than the intermediate-aged stars. The centroid of the young stars is offset by $\approx 34^{\prime \prime}$, and they have a more centrally-concentrated and flattened spatial distribution. Also, the major axis of the young stars is offset from that of the intermediate-aged stars by $\approx 45^{\circ}$.

The presence of such surprisingly young stars in Fornax raises the question of whether there remains an undetected reservoir of gas in this dSph. Could there be significant amounts of neutral gas that is not seen because of its large, possibly flattened, spatial extent? Does Fornax have $\mathrm{H}$ I in a disk lying outside the optical radius similar to what Carignan et al. (1998) have found recently for the Sculptor dSph? Could a significant amount of gas be hidden in a diffuse ionized component that is kept excited by the young massive stars, hot stellar remnants, or the meta-galactic UV background? These are some of the exciting questions that our survey has generated.

\section{Our Deep Photometric Survey}

The new CMD presented in Fig. 1 shows the preliminary results of our deep photometric survey of Fornax done with the prime focus imager on the CTIO 4-m Telescope. For comparison, we show the CMD we obtained for the Carina $\mathrm{dSph}$ with the same instrument. The Fornax CMD contains $~ 56000$ stars and is based on total integration times of $2.5 \mathrm{hrs}$ in each filter (roughly $25 \%$ of our total data) for an area approximately $15^{\prime} \times 15^{\prime}$ in size. The center of this area is located $\sim 10$ arcmin north-west of the center of the Fornax dSph, and thus we sample a wide range in galactocentric radii. Photometric reduction of the data with DAOPHOT and ALLFRAME software is in progress. Eventually our CMD will go more than 1.5 mag deeper than that shown in Fig. 1.

\section{Findings from the CMDs}

From comparison of the Fornax and Carina CMDs with Padova theoretical isochrones (Bertelli et al. 1994), several points emerge. First, no significant "gaps" occur in the main-sequence turnoff region of Fornax's CMD compared to Carina's CMD, which means that Fornax's star-formation rate was more continuous than episodic. This probably reflects the fact that Fornax is a more massive galaxy, and hence its star-formation rate is not as easily disrupted by stellar winds and supernovae. (For comparison, $M_{V}$ of Fornax is 4.4 mag brighter than Carina, and the total mass of Fornax is at least 10 times larger than Carina as inferred from modelling of their stellar velocity dispersions.) 

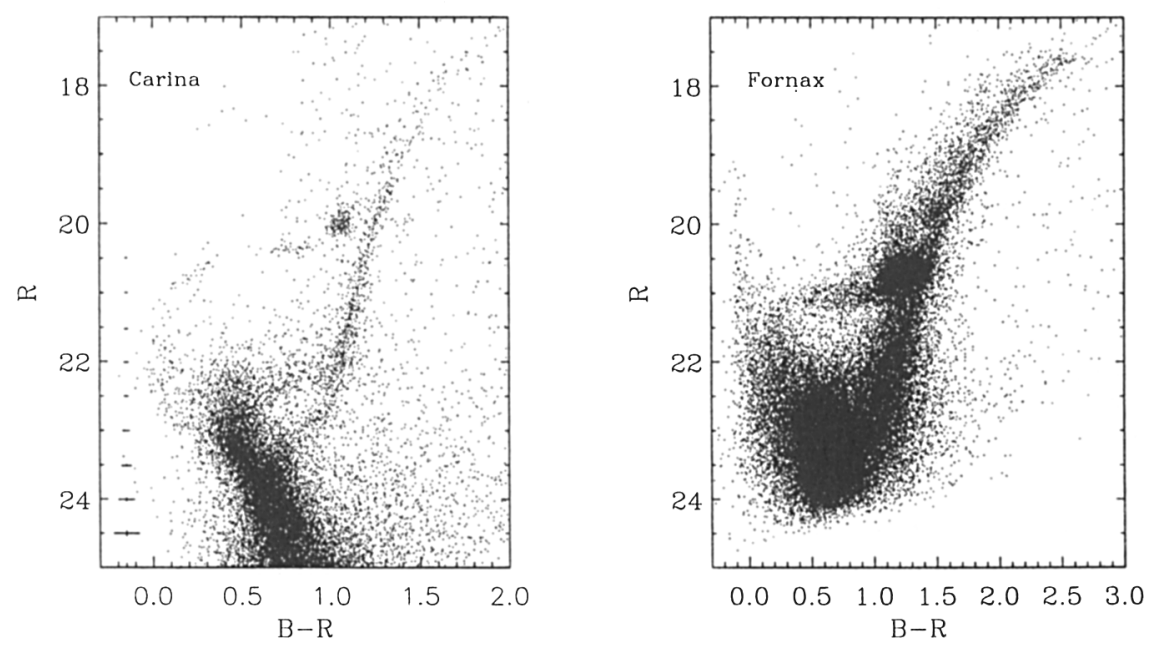

Figure 1. CMDs of the Carina and Fornax dSph galaxies (SmeckerHane et al. 1998a and 1998b).

Second, the sharp discontinuity in the luminosity function of Fornax's main sequence at $R \approx 22.7, B-R \approx 0.6$ suggests that its star-formation rate decreased by a significant amount $\sim 2$ Gyr ago. However, star-formation did not cease. The "blue plume" of main-sequence stars $(R<22$ and $B-R \approx 0)$ proves that Fornax has a sprinkling of surprisingly young ( 100 to $400 \mathrm{Myr}$ old), relatively metal-rich $(Z \approx 0.004)$, stars. This combination of age and metallicity reproduces the shape of the blue plume and its complementary RGB/AGB, but until metallicities are determined via spectroscopy the absolute ages cannot be determined very accurately. The total range in ages is impressive: $\sim 100 \mathrm{Myr}$ to 14 Gyr. Star formation in Fornax occurred over most of the age of the Universe.

Third, the clump horizontal branch (HB) containing intermediate-aged (3$10 \mathrm{Gyr}$ ) stars has significant substructure that will be interesting to compare carefully with theoretical tracks. The CMD also contains a $\mathrm{HB}$ of bluer and fainter stars, more typical of a globular cluster HB, that undoubtedly contains some of Fornax's oldest stars. The spread in colors of the RGB stars, and the spreads in colors and magnitudes of the HB stars, suggest that the metallicities of Fornax stars probably span the range from $Z=0.0004$ to $Z=0.008$ $(-2 \leq[\mathrm{Fe} / \mathrm{H}] \leq-0.7)$, which roughly agrees with previous work done on Fornax (Buonanno et al. 1985; Beauchamp et al. 1995).

\section{Discussion}

We find that the Fornax $\mathrm{dSph}$ has very complex stellar populations that span an impressive range of ages and metal abundances, even more than those of the Carina and Leo II dSphs. Leo II has stars from 7 to $14 \mathrm{Gyr}$ in age (Mighell \& Rich 1996), and Carina has stars ranging from $~ 2.5$ to 14 Gyr in age but with a noticeable lack of stars in the 7 to $11 \mathrm{Gyr}$ interval. Clearly each of these 
dSphs is unique. Quantifying their differences is the first step to understanding why they evolved so differently and what their complex behavior tells us about galaxy formation, in general.

\section{References}

Beauchamp, D., Hardy, E., Suntzeff, N.B., Zinn, R. 1985, A\&A, 145, 97

Bertelli, G., et al. 1994, A\&AS, 106, 275

Buonanno, R., et al. 1985, A\&A, 145, 97

Carignan, C., et al. 1998, AJ, 116, 1690

Irwin, M., Hatzidimitriou, D. 1995, MNRAS, 277, 1354

Mighell, K.J., Rich, R.M. 1996, AJ, 111, 777

Smecker-Hane, T.A., Stetson, P.B., Hesser, J.E., VandenBerg, D. 1998a, in prep.

Smecker-Hane, T.A., Stetson, P.B., Hesser, J.E., VandenBerg, D. 1998b, in prep.

Stetson, P.B., Hesser, J.E., Smecker-Hane, T.A. 1998, PASP, 110, 533 